\title{
Nicotinamide Phosphoribosyl Transferase a Reliable Marker of Progression in Cervical Dysplasia
}

\author{
MOIZ VORA ${ }^{1}$, LUBNA A. ALATTIA $^{1}$, JUNAID ANSARI ${ }^{2}$, MENCHU ONG $^{1}$, JAMES COTELINGAM $^{1}$, \\ DOMENICO COPPOLA ${ }^{3}$ and RODNEY SHACKELFORD ${ }^{1}$ \\ ${ }^{1}$ Department of Pathology and Translational Pathobiology, LSU Health, Shreveport, LA, U.S.A.; \\ ${ }^{2}$ Department of Molecular and Cellular Physiology, LSU Health Sciences Center, Shreveport, LA, U.S.A.; \\ ${ }^{3}$ Department of Anatomic Pathology, H. Lee Moffitt Cancer Center and Research Institute, Tampa, FL, U.S.A.
}

\begin{abstract}
Background/Aim: Nicotinamide phosphoribosyl transferase (Nampt) catalyses the rate-limiting step of the mammalian nicotinamide adenine dinucleotide (NAD) salvage pathway. Nampt is highly expressed in several epithelial and mesenchymal neoplasms, where is promotes cell-cycle progression ans chemotherapy resistance. To our knowledge, alterations in Nampt expression have not been examined in cervical intraepithelial neoplasia (CIN) or squamous cell carcinoma (SCC). Materials and Methods: We performed immunohistochemical analysis for Nampt using tissue microarrays on 14 samples of benign cervical squamous epithelium and 15 CIN I, 15 CIN II, and 13 samples of CIN III. The SCCs included 5 low-grade, 67 intermediate-grade, and 81 high-grade tumors. Results: Nampt levels increased with increased CIN grades were compared to benign cervical squamous epithelium. Similarly, Nampt levels increased with increasing SCC grade. Conclusion: Nampt expression is a reliable marker of progression in cervical dysplasia and SCC.
\end{abstract}

Cervical cancer is the third most prevalent gynecologic malignancy worldwide and more than $85 \%$ of the disease burden occurs in developing nations (1-3). Invasive squamous cell carcinomas arising from the surface epithelium are the most common (4). The earliest histological precursor of SCC is low-grade squamous dysplasia (CIN I) characterized by nuclear enlargement, hyperchromasia, and irregular nuclear contours. Higher

Correspondence to: Rodney Shackelford, LSU Health Shreveport, Department of Pathology, 1501 Kings HWY, Shreveport, LA 71130, U.S.A. Tel: +1 3186751228, Fax: +1 3186758395, e-mail: rshack@1suhsc.edu

Key Words: Nicotinamide phosphoribosyl transferase, cervical dysplasia, squamous cell carcinoma. grades of dysplasia (CIN II-III) are accompanied by increased nuclear-cytoplasmic ratios, coarsening of chromatin, increased mitotic activity, and extension of dysplastic keratinocytes to the upper-third of the cervical epithelium (7). The majority of low-grade lesions regress spontaneously, whereas higher-grade dysplasia further progresses to in situ carcinoma, which yet may be curable by surgical management. However, invasive or recurrent disease carries a poor prognosis such that the early detection of cervical cancer and its preceding lesions is vital for optimal patient outcomes $(5,6)$.

Cervical SCC development is closely associated with persistent high-risk human papilloma virus (HPV) infection $(8,9)$. The oncogenic potential of HPV comes largely from two viral proteins, E6 and E7, that bind many cellular targets, including the $\mathrm{p} 53$ and $\mathrm{pRb}$ proteins resulting in their degradation (10-13). E6 and E7 function coordinately to ablate apoptotic and cell-cycle checkpoint responses, increases telomerase activity, and increases somatic mutations, all of which promote carcinogenesis (6, 10-15). HPV infection also promotes carcinogenesis by increasing STAT3 protein kinase expression and activity (16). Not surprisingly, CIN shows increased STAT3 protein with increasing CIN grade and in cervical SCC compared to benign cervical epithelium (17). STAT3 activity induces higher expression of nicotinamide phosphoribosyl transferase (Nampt), an enzyme that catalyses the rate-limiting step of nicotinamide adenine dinucleotide $\left(\mathrm{NAD}^{+}\right)$synthesis (18). Nampt is elevated in a number of human malignancies, including gliomas, melanoma, prostate, breast, ovarian, thyroid, and colorectal carcinomas (19-26). High Nampt expression promotes cell growth and survival, DNA synthesis, mitochondrial biogenesis, angiogenesis, contributing to malignant progression (27). Recently Nampt protein expression was found to be increasingly elevated with higher grades (dedifferentiation) of leiomyosarcomas and aggressive histologic subtypes of rhabdomyosarcomas (28). 
Table I. IHC: Relative Nampt staining in the tissue microarrays comparing benign cervical tissue to CIN I-III and grades I, II, and III SCC.

\begin{tabular}{lccc}
\hline Tissue Type & Sample number & Average nampt IHC score & SEM \\
\hline Benign cervical squamous epithelium & 14 & 1.57 & 0.14 \\
CIN I & 11 & 3.45 & 0.49 \\
CIN II & 9 & 3.86 & 1.02 \\
CIN III & 8 & 4.91 & 0.76 \\
SCC Grade I & 5 & 4.00 & 0.55 \\
SCC Grade II & 56 & 4.91 & 0.32 \\
SCC Grade III & 53 & 6.43 & 0.37 \\
Benign cervical columnar epithelium & 13 & 1.77 & 0.23 \\
Adenocarcinoma & 7 & 7.71 & 0.95 \\
\hline
\end{tabular}

IHC: Immunohistochemistry; SEM: standard error of the mean.

Based upon these observations we hypothesized that Nampt expression would be increasingly elevated with higher grades of in situ cervical dysplasia and squamous cell carcinoma as compared to benign cervical epithelium. Herein we performed a semi-quantitative immunohistochemical analysis of Nampt expression utilizing a tissue microarray.

\section{Materials and Methods}

Tissue microarrays (TMAs), catalog numbers CIN481 and CxC1501, were purchased from US Biomax, Inc. (Rockville, MD, USA). Together the TMAs contained the following: 14 samples of benign squamous epithelium, 15 CIN I, 15 CIN II, and 13 samples of CIN III. Tissue samples of squamous cell carcinoma included 5 low grade, 67 intermediate grade, and 81 high-grade tumors. Additionally 13 cores of benign endocervical columnar epithelium and 7 samples of endocervical adenocarcinoma were represented in the microarrays. The biopsy diameters of each core were $1.5 \mathrm{~mm}$ and $1.1 \mathrm{~mm}$ in the CIN481 and CxC1501 TMAs respectively.

NAMPT immunohistochemistry (IHC). The concentration of primary Nampt antibody was optimized to normal kidney as control tissue. The staining of the TMA was performed in the Tissue Core Histology Lab Facility at the Moffitt Cancer Center. The microarray slides were stained using a Ventana Discovery XT automated system (Ventana Medical Systems, Tucson, AZ, USA) as per the manufacturer's protocol with proprietary reagents. Briefly, slides were deparaffinized on the automated system with EZ Prep solution (\#950-100; Ventana Medical Systems). The heat-induced antigen retrieval method was used in Cell Conditioning 1 (\#950-124; Ventana Medical Systems). Mouse monoclonal antibody to human Nampt (\#ALX-804-717; Enzo life Sciences, Plymouth Meeting, PA, USA) was used at a 1:1,000 concentration in Dako antibody diluent (\#S0809; Dako, Carpenteria, CA, USA) and incubated for $60 \mathrm{~min}$. The Ventana anti-mouse or rabbit secondary antibodies were used for $16 \mathrm{~min}$. The detection system used was the Ventana OmniMap kit. Slides were then dehydrated and cover-slipped as per standard laboratory protocol.
Evaluation of NAMPT staining. Relative Nampt protein expression was determined as immunostain intensity scored on a 0-3 scale as follows: no staining as 0 , light staining as 1 , moderate staining as 2 , and heavy staining as 3 . The percentage of cells stained was measured, with no detectable staining as $0,1-33 \%$ as $1,34-66 \%$ as 2 and $67-100 \%$ as 3 . The final IHC score was the product of the percentage of cells stained multiplied by the intensity score, allowing for a maximal score of 9 and a minimal score of 0 . Nuclear and cytoplasmic Nampt staining was seen in all tissue samples examined, although at low levels in benign squamous and columnar epithelium. We therefore measured and quantified Nampt staining in the nuclear and cytoplasmic compartments. Since even experienced pathologists can disagree on diagnosing the lower dysplasia grade, all cores on the TMA slide were reviewed by three pathologists and to ensure that the histologic CIN grading was exact and accurate.

Statistical analysis. The standard error of the mean (SEM) IHC score was calculated by using the standard deviation for the staining scores of each tumor type and dividing this number by the square root of the sample size.

\section{Results}

Following IHC processing, due to tissue loss we were left with 14 samples of benign squamous epithelium and 13 cases of benign endocervical columnar epithelium. Of the CIN tissue 11 CIN I, 9 CIN II, and 8 III samples cases were available for analysis. Of the invasive carcinoma cases 5 low grade, 56 intermediate grade, and 53 high-grade SCC, and 7 cervical adenocarcinomas tissue cores were available for analysis. A number tissue samples on the TMA were not analyzed as they were unrelated to purpose of the present study (adenosquamous carcinoma-7 cases, $\mathrm{CxC1501)}$ or insufficiently represented (hyperplasia-2 cases $\mathrm{CxC} 1501$, mucinous adenocarcinoma-1 case CIN481). Examples of Nampt IHC of benign, dysplastic, and malignant tissues are shown in Figure 1. The number of cases examined and the quantified IHC scores for each tissue type are given in Table I. 

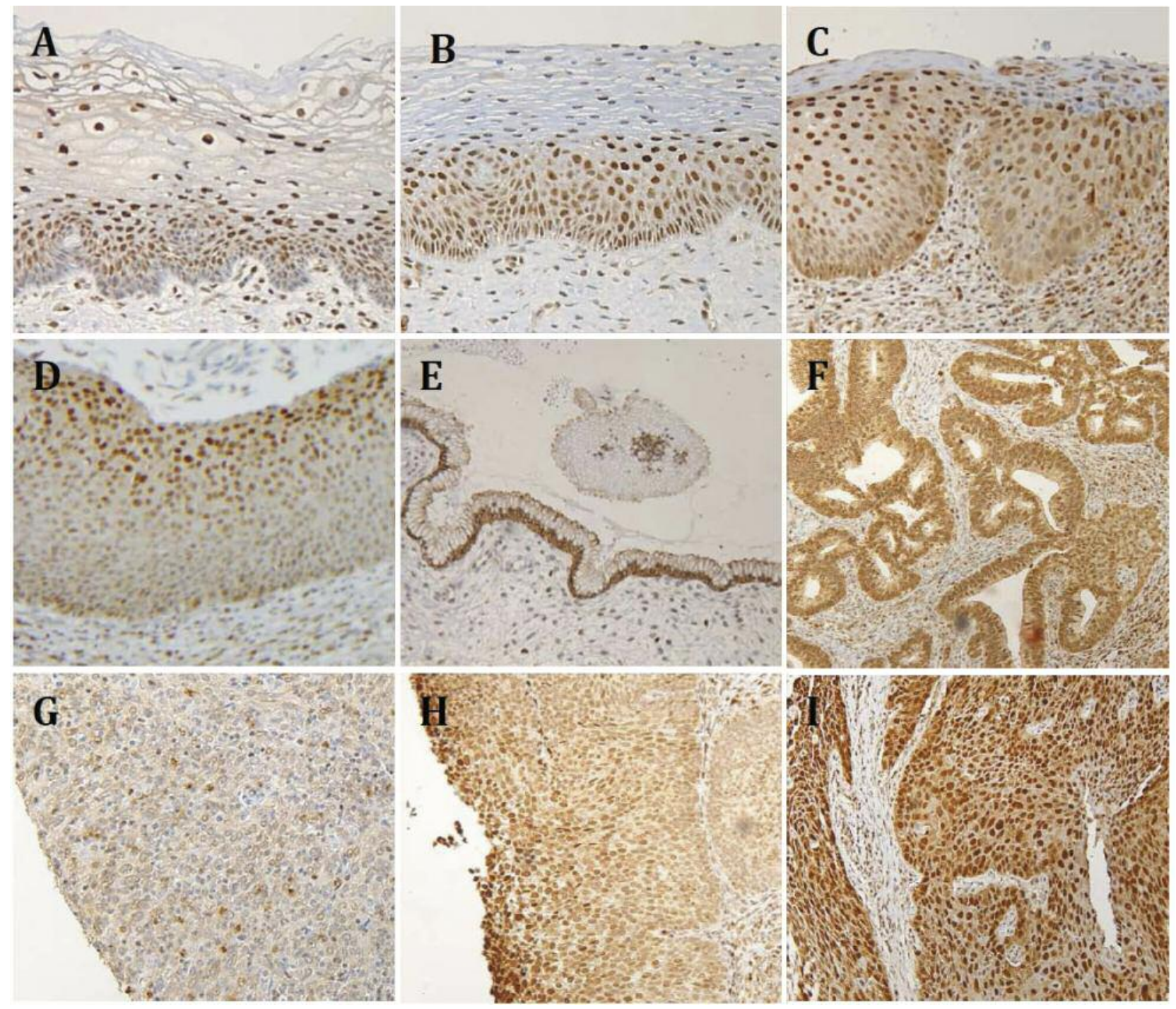

Figure 1. High- and low-power views of Nampt IHC of benign cervix (A), CIN I (B), CIN II (C), CIN III (D), benign columnar epithelium (E), adenocarcinoma $(F)$, grade I SCC $(G)$, grade II SCC $(H)$, and grade III SCC (I). Panels A-D are high-power views (200x) and E-I are low power views $(100 \times)$.

\section{Discussion}

In cervical biopsies CIN is commonly analyzed by the microscopic examination of properly oriented hematoxylin and eosin stained tissue sections (29-33). Commonly, these analyses are supplemented by p16 immunohistochemistry that is a sensitive marker for HPV E7 protein expression (29, 30). Cushieri et al. (30) reviewed nine studies including 2,178 cases and found p16 expression in $7 \%$ of benign cervical epithelium, and in 54\%,86\%, and $96 \%$ of CIN I-III cases, respectively. Other less often used protein markers for CIN include Ki-67, telomerase, p63, and E-cadherin (29-33).
Here we show that Nampt is increased in a grade-dependent manner in CIN and cervical SCC. Nampt catalyses the ratelimiting step of $\mathrm{NAD}^{+}$synthesis and Nampt levels and enzymatic activity are the main determinants of cellular $\mathrm{NAD}^{+}$levels $(27,33,34) . \mathrm{NAD}^{+}$is both a vital energy source and signal transduction molecule that is rapidly degraded in malignant cells, hence Nampt protein levels and Nampt-mediated $\mathrm{NAD}^{+}$synthesis is increased in many malignancies (19-27, 35). Nampt is most highly expressed in active dividing cells and higher Nampt expression confers a worse prognosis in prostate and gastric cancers and increases chemotherapy resistance in gastric cancer $(22,25,27)$. 
Additionally, Nampt is more highly expressed with increasing Fuhrman grade in renal cell carcinoma, and in higher histiologic grades of oral squamous cell carcinoma and leiomyosarcomas $(28,36,37)$.

In the present case of invasive cervical SCC we were also able to demonstrate that Nampt i) is a marker for cervical dysplasia in CIN which increases in a grade-dependent manner, ii) increases with higher grades of cervical SCC, and iii) is highly expressed in cervical adenocarcinoma compared to benign cervical columnar epithelium (Table I and Figure 1) $(19-32,38)$. Also since p16 is expressed in only $54 \%$ of CIN I, and we found Nampt expression to be doubled in CIN I as opposed to benign squamous epithelium (Table I), our study suggests that Nampt IHC may be useful in CIN analysis in these situations. This is noteworthy particularly since $\mathrm{NAD}^{+}$synthesis is obligatory for cell survival and growth $(27,29,30,39)$. Further investigation is needed to ascertain the possible use of Nampt IHC in the analysis and diagnosis of CIN.

\section{Conflicts of Interest}

The Authors report no conflicts of interest.

\section{Acknowledgements}

The Authors thank the Histology Section of the Tissue Core at the Moffitt Cancer Center and Research Institute for the support in performing the IHC stains.

\section{References}

1 Ferlay J, Shin HR, Bray F, Forman D, Mathers C and Parkin DM: Estimates of worldwide burden of cancer in 2008: GLOBOCAN 2008 I. J. Cancer J Int Cancer 127: 2893-2928, 2010.

2 Siegel RL, Miller KD and Jemal A: Cancer statistics 2017. CA Cancer J Clin 67: 7-30, 2017.

3 Maguire R, Kotronoulas G, Simpson M and Paterson M: A systematic review of the supportive care needs of women living with and beyond cervical cancer. Gynecol Oncol 136: 478-490, 2015.

4 Mathew A and George PS: Trends in incidence and mortality rates of squamous cell carcinoma and adenocarcinoma of cervixworldwide. Asian Pac J Cancer Prev 10: 645-650, 2009.

5 Diaz-Padilla I, Monk BJ, Mackay HJ and Oaknin A: Treatment of metastatic cervical cancer: future directions involving targeted agents. Crit Rev Oncol Hematol 85: 303-314, 2013.

6 Mishra GA, Pimple SA and Shastri SS: Prevention of Cervix Cancer in India. Oncology 91: 1-7, 2016.

7 Richart RM: The patient with an abnormal Pap smear screening techniques and management. N Engl J Med 302: 332334, 1980.

8 Walboomers JM, Jacobs MV, Manos MM, Bosch X, Kummer A, Shah K, Snuders P, Peto J, Meijer C and Munos N: Human papillomavirus is a necessary cause of invasive cervical cancer worldwide. J Pathol 189: 12-19, 1999.
9 Herfs $\mathrm{M}$ and Crum CP: Cervical cancer: squamocolumnar junction ablation - tying up loose ends? Nat Rev Clin Oncol 12: 378-380, 2015.

10 White EA and Howley PM: Proteomic approaches to the study of papilloma virus host interactions. Virology 435: 57-69, 2013.

11 Werness BA, Levine AJ and Howley PM: Association of human papillomavirus types 16 and 18 E6 proteins with p53. Science 248: 76-79, 1990.

12 Boyer SN, Wazer DE and Band V: E7 protein of human papilloma virus-16 induces degradation of retinoblastoma protein through the ubiquitinproteasome pathway. Cancer Res 56: 4620-4624, 1996.

13 Huh KW, DeMasi J, Ogawa H, Nakatani Y, Howley P and Münger K: Association of the human papillomavirus type 16 E7 oncoprotein with the $600-\mathrm{kDa}$ retinoblastoma protein-associated factor, p600. Proc Natl Acad Sci USA 102: 11492-11497, 2005.

14 Spardy N, Duensing A, Hoskins EE, Wells SI and Duensing S: HPV-16 E7 reveals a link between DNA replication stress, fanconi anemia D2 protein, and alternative lengthening of telomere-associated promyelocytic leukemia bodies. Cancer Res 68: 9954-9963, 2008.

15 Shackelford RE, Kaufmann WK and Paules RS: Cell cycle control, checkpoint mechanisms, and genotoxic stress. Environ Health Perspect 107(Suppl 1): 5-24, 1999.

16 Shukla S, Shishodia G, Mahata S, Hedau S, Pandey A, Bhambhani S, Batra S, Basir S, Das BC and Bharti AC: Aberrant expression and constitutive activation of STAT3 in cervical carcinogenesis: implications in high-risk human papillomavirus infection. Mol Cancer 9: 282, 2010.

17 Yang SF, Yuan SS, Yeh YT, Hung SC, Wu MT, Su JH and Chai CY: Positive association between STAT3 and Ki-67 in cervical intraepithelial neoplasia. Kaohsiung J Med Sci 22: 539-546, 2006.

18 Nowell MA, Richards PJ, Fielding CA, Ognjanovic S, Topley $\mathrm{N}$, Williams AS, Bryant-Greenwood $\mathrm{G}$ and Jones SA: Regulation of pre-B cell colony enhancing factor by STAT-3dependent inter-leukin-6 trans-signaling: implications in the pathogenesis of rheumatoid arthritis. Arthritis Rheum 54: 20842095, 2006.

19 Reddy PS, Umesh S, Thota B, Tandon A, Pandey P, Hegde AS, Balasubramaniam A, Chandramouli BA, Santosh V, Rao MR, Kondaiah P and Somasundaram K: PBEF1/NAmPRTase/Visfatin: a potential malignant astrocytoma/glioblastoma serum marker with prognostic value. Cancer Biol Ther 7: 663-668, 2008.

20 Rodney E Shackelford, Bui M, Coppola D and Hakam A: Overexpression of nicotinamide phosphoribosyltransferase in ovarian cancers. Int J Clin Exp Pathol 3: 522-527, 2013.

21 Shackelford R, Hirsh S, Henry K, Abdel-Mageed A, Kandil E and Coppola D: Nicotinamide phosphoribosyltransferase and SirT3 expression are increased in well-differentiated thyroid carcinomas. Anticancer Res 33: 3047-3052, 2013.

22 Wang B, Hasan, MK, Alvarado E, Yuan H, Wu H and Chen WY: NAMPT overexpression in prostate cancer and its contribution to tumor cell survival and stress response. Oncogene 30: 907921, 2011.

23 Maldi E, Travelli C, Caldarelli A, Agazzone N, Cintura S, Galli U, Scatolini M, Ostano P, Miglino B, Chiorino G, Boldorini R and Genazzani AA: Nicotinamide phosphoribosyltransferase (NAMPT) is over-expressed in melanoma lesions. Pigment Cell Melanoma Res 26: 144-146, 2013. 
24 Kim JG, Kim EO, Jeong BR, Min YJ, Park JW, Kim ES, Namgoong IS, Kim YI and Lee BJ: Visfatin stimulates proliferation of MCF-7 human breast cancer cells. Mol Cells 30: 341-345, 2010.

25 Bi TQ, Che XM, Liao XH, Zhang DJ, Long HL, Li HJ and Zhao W: Overexpression of Nampt in gastric cancer and chemopotentiating effects of the Nampt inhibitor FK866 in combination with fluorouracil. Oncol Rep 26: 1251-1257, 2011.

26 Huang WS, Chen CN, Sze CI and Teng CC: Visfatin induces stromal cell derived factor- 1 expression by $\beta 1$ integrin signaling in colorectal cancer cells. J Cell Physiol 228: 1017-1024, 2013.

27 Shackelford RE, Mayhall K, Maxwell NM, Kandil E and Coppola D: Nicotinamide phosphoribosyltransferase in malignancy: a review. Genes Cancer 4: 447-456, 2013

28 Vora M, Ansari J, Shanti RM, Veillon D, Cotelingam J, Coppola D and Shackelford RE: Increased nicotinamide phosphoribosyltransferase in rhabdomyosarcomas and leiomyosarcomas compared to skeletal and smooth muscle tissue. Anticancer Res 36: 503-507, 2016.

29 Silva DC, Gonçalves AK, Cobucci RN, Mendonça RC, Lima PH and Cavalcanti G Júnior: Immunohistochemical expression of p16, Ki-67 and p53 in cervical lesions - A systematic review. Pathol Res Pract 7: 723-729, 2017.

30 Cuschieri $\mathrm{K}$ and Wentzensen N: Human papillomavirus mRNA and p16 detection as biomarkers for the improved diagnosis of cervical neoplasia. Cancer Epidemiol Biomarkers Prev 17: 25362545,2008

31 Sui W, Ou M, Dai Y, Chen J, Lan H, Yan Q and Huang H: Gain of the human telomerase RNA gene TERC at $3 q 26$ is strongly associated with cervical intraepithelial neoplasia and carcinoma Int J Gynecol Cancer 19: 1303-1306, 2009.

32 Ancuţa E, Ancuţa C, Cozma LG, Iordache C, AnghelacheLupaşcu I, Anton E, Carasevici E, and Chirieac R: Tumor biomarkers in cervical cancer: focus on $\mathrm{Ki}-67$ proliferation factor and E-cadherin expression. Rom J Morphol Embryol 50: 413-418, 2009.
33 Shirendeb U, Hishikawa Y, Moriyama S, Win N, Thu MM, Mar KS, Khatanbaatar G, Masuzaki $\mathrm{H}$ and Koji T: Human papillomavirus infection and its possible correlation with p63 expression in cervical cancer in Japan, Mongolia, and Myanmar. Acta Histochem Cytochem 42: 181-190, 2009.

34 Rongvaux A, Shea RJ, Mulks MH, Gigot D, Urbain J, Leo O and Andris F: Pre-B-cell colony-enhancing factor, whose expression is up-regulated in activated lymphocytes, is a nicotinamide phosphoribosyltransferase, a cytosolic enzyme involved in NAD biosynthesis. Eur J Immunol 32: 3225-3234, 2002.

35 Chiarugi A, Dölle C, Felici R and Ziegler M: The NAD metabolome-a key determinant of cancer cell biology. Nat Rev Cancer 12: 741-752, 2012.

36 Shackelford R, Abdulsattar J, Wei E, Cotelingam J, Coppola D and Herrera G: Increased nicotinamide phosphoribosyltransferase and cystathionine $\beta$-synthase in renal oncocytomas, renal transitional cell carcinoma and renal clear cell carcinoma. Anticancer Res 37: 3423-3427, 2017.

37 Patel S, Ansari J, Meram A, Abdulsattar J, Cotelingam J, Coppola D, Ghali G and Shackelford R: Increased nicotinamide phosphoribosyltransferase and cystathionine-beta-synthase in oral cavity squamous cell carcinomas. Internat $\mathrm{J}$ of Clin and Exper Med 10: 702-707, 2017.

38 Revollo JR, Grimm AA and Imai S: The NAD biosynthesis pathway mediated by nicotinamide phosphoribosyltransferase regulates Sir2 activity in mammalian cells. J Biol Chem 279: 50754-50763, 2004.

39 Kanthiya K, Khunnarong J, Tangjitgamol S, Puripat N and Tanvanich S: Expression of the p16 and Ki67 in cervical squamous intraepithelial lesions and cancer. Asian Pac J Cancer Prev 17: 3201-3206, 2016. 